\title{
STUDY AND ANALYSIS OF RF MEMS SHUNT SWITCH FOR RECONFIGURABLE ANTENNA
}

\author{
H. Divya \\ PG Scholar, Electronics and Communication Engineering, Saveetha Engineering College, Tamil Nadu, India
}

\begin{abstract}
$R F$ MEMS switches can be used to achieve reconfigurability of various $R F$ systems and in particularly for miniaturized antenna structures. In case of micro machined antennas, which involve low voltage signals, RF MEMS switches with low actuation voltage are required for achieving reconfigurability. The capacitive shunt switch derives its switching property from the significant difference of its capacitance in the up-state and down-state. The actuation voltage of RF MEMS switches mainly depends on the spring constant of the switch membrane. In this project a low actuation voltage capacitive shunt switch suitable to be used along with micro machined antennas is designed. A process flow for the fabrication is designed and simulated using Intellisuite. The electromechanical analysis results are presented and compared with that of a fixed-fixed flexure based switch membrane to establish the low actuation voltage characteristics of the proposed design
\end{abstract}

Keywords: RF MEMS, Reconfigurable antenna, shunt switch

\section{INTRODUCTION}

Radio Frequency (RF) systems designed for single predefined mission antennas with fixed characteristics such as frequency band, radiation pattern, polarization, and gain. Applications such as cognitive radio system, Multiple-input multiple-output (MIMO) channels and satellite communication need antenna with the reconfigurable parameters [1].

Reconfiguring of antenna is achieved through changing its frequency, polarization or radiation characteristics by using Radio-Frequency Micro Electro Mechanical Systems (RFMEMS), PIN Diodes, Varactors and FETs .Even though they have slow switching speed, unlike the other switching devices, RF MEMS switches are mechanical switches electronically controlled and have near zero power consumption, low insertion loss and high isolation. This mechanical movement is achieved using electrostatic, piezoelectric, magneto static or thermal actuation. Even though electrostatic method requires a high actuation voltage it is the most prevalent one due to its near zero power consumption, small electrode size, thin layers and short switching time. On the basis of contact mechanism and the position with respect to transmission line [2], RF MEMS switch can be classified as capacitive or ohmic and series or shunt .The shunt RF MEMS switches are capacitive in nature where the mechanical movement of the switch membrane introduces a variable capacitance between the signal line and the ground.

The capacitive RF MEMS switches used along with CPW and integrated with RF circuits to achieve reconfigurability requires low actuation voltages. The actuation voltage of the RF MEMS switches [3] can be reduced so as to make it compatible with the associated control circuits by varying the spring constant, actuation area or the gap between the switch membrane and the actuation electrode. Lowering the spring constant by using different geometric structures for the switch membrane can reduce the spring constant and the actuation voltage. In this paper an RF MEMS capacitive switch [4] operating at a low actuation voltage of $0.35 \mathrm{Volt}$ is presented. A process flow has been designed for the proposed switch and tested in IntelliFab. The mask files for the fabrication are designed and results of electromechanical analysis of the proposed design are presented.

\section{SYSTEM DESCRIPTION}

A reconfigurable antenna is one which alters its radiation, polarization and frequency characteristics by morphing its physical structure. Reconfigurable antennas with the ability to radiate more than one pattern at different frequencies are necessary in radar and modern communication systems. Many reconfigurable antennas concentrate on changing operating frequency while maintaining their radiation characteristics. However, the ability to change the radiation patterns while maintaining operating frequency could greatly enhance system performance.

Manipulation of an antenna's radiation pattern can be used to avoid noise sources or intentional jamming, improve security by directing signals only toward intended users, serve as a switched diversity system, and expand the beam steering capabilities of large phased arrays.

RF MEMS switches are employed in many ways to achieve reconfigurability. The first is to change the shape of the 
effective radiating structure to alter the pattern or the frequency of operation. The second method employs RF MEMS to mechanically actuate the antenna [5], and change the orientation of the antenna with respect to the substrate or another radiating structure. The third method employs RF MEMS capacitive switches to modify the impedance of the antenna, which changes the resonant frequency of the radiating antenna. The fourth employs MEMS phase shifters.

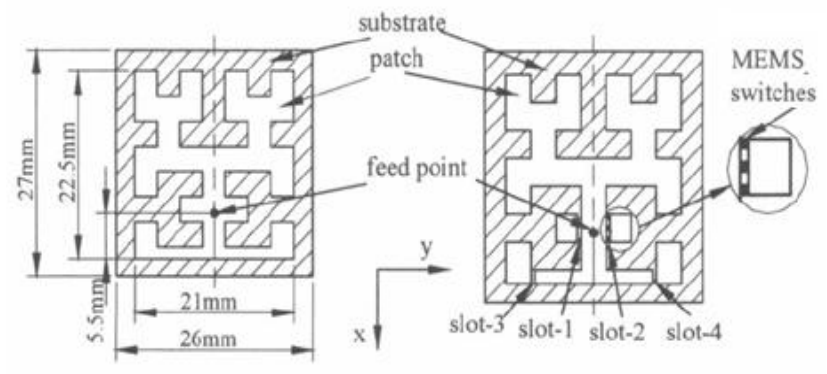

(a)

(b)

Fig.1 (a) Layout of the original patch antenna, (b) Antenna being reconfigured by use of MEMS switches

\section{RF MEMS SHUNT SWITCH}

Radio Frequency Micro-electromechanical Systems (RF MEMS) are becoming a popular technology for many RF applications. Although MEMS have been developed since the 1970s for sensors, accelerometers, gas chromatographs, and other sensor devices, it did not get much attention from the RF and Microwave frequency community for a long time.

MEMS switches use the same principle as the simple mechanical moving switch, like basic single pole single through (SPST) [6]. The beam moves mechanically either to make an open or short circuit. The difference is that it is miniaturized and works at RF and Microwave frequencies. Shows a basic MEMS cantilever switch with a separate actuation electrode.

When a DC voltage is applied between the actuation electrode and the suspended beam, an electrostatic force will develop and pull the beam down. This will create a contact between the signal in and signal out electrodes. Depending on the configuration the contact can be two types: DC contact and capacitive contact.

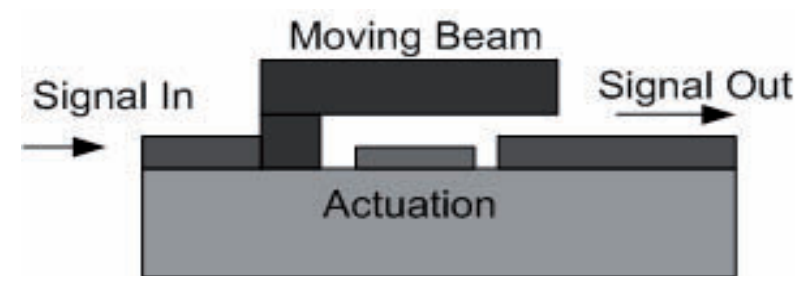

Fig 2 RF shunt switch

\section{OPERATING PRINCIPLES:}

Various kinds of basic RF MEMS switches are shown in Figures $1.3-1.6$. From an electrical contact point of view the MEMS switches can be two types: a) Capacitive contact, and b) DC contact.

A dielectric is deposited on top of the bottom electrode of the capacitive contact switch as shown in Figure 1.3 and 1.5. When the suspended beam is in the upstate the capacitance is in the range of $\mathrm{fF}$.

When a DC actuation voltage is applied between the actuation electrode and the suspended beam, the suspended beam will move downward and collapse on the bottom electrode. This will increase the capacitance in the range of $\mathrm{pF}, 20-100$ times higher than the upstate capacitance.

The upstate capacitance depends mainly on the initial gap. The downstate capacitance depends on the dielectric thickness, dielectric constant etc. $\mathrm{SiO} 2, \mathrm{Si} 3 \mathrm{~N} 4, \mathrm{TiO} 2$ can be used as dielectric for RF MEMS switches. The capacitive contact switch is suitable for high frequency application. At low frequency (DC), the impedance always will be very high for a capacitive switch, whatever the capacitance.

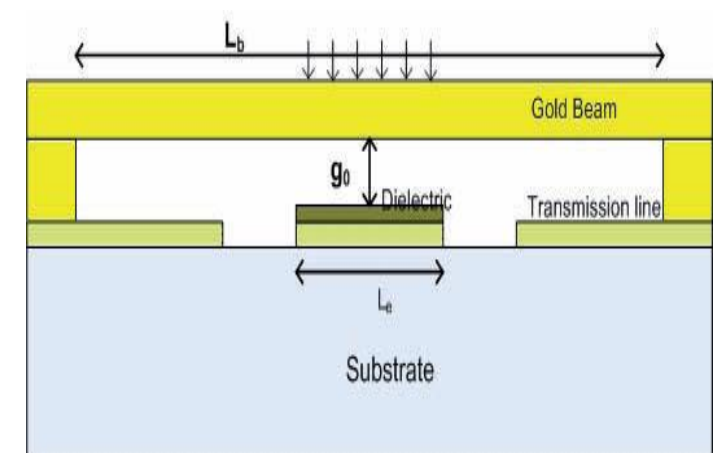

Fig 3 capacitive contact switch

\section{PERFORMANCE}

RF MEMS switches have extremely low insertion loss during the on-state and very high isolation during the-off state. They are very suitable for operation at RF and Microwave frequency region.

They are extremely linear and have very low intermodulation products. They consume very little power during actuation and can provide a very high capacitance ratio compared to their counterparts. The performance of RF MEMS switches compared to their counter parts, PIN diode and FET switches. 


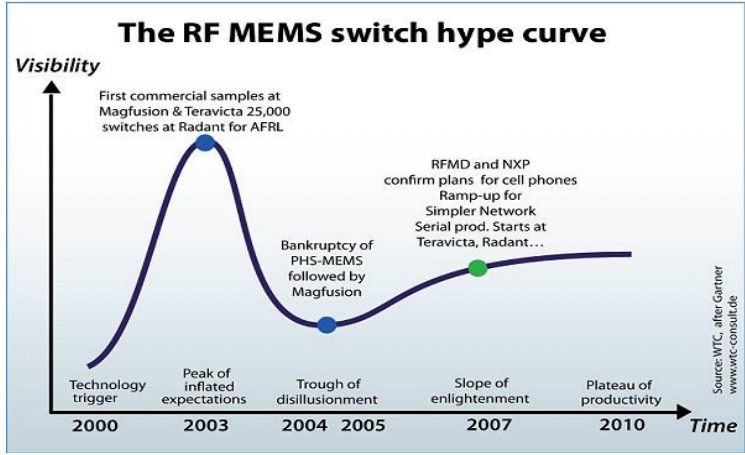

Fig 4 RF MEMS switch hype curve

\section{APPLICATIONS}

RF MEMS have very good RF performance in the RF and Microwave region. DC contact switches provide very good insertion loss and isolation at low frequency from DC to several tens of $\mathrm{GHz}$.

Capacitive contact switches [7] provide very good insertion loss and isolation at higher frequency from $3-4 \mathrm{GHz}$ to 100 GHz.

The power consumption of RF MEMS switches is very low or close to zero, so it can be used in portable systems where the battery life time is very important.

The cut-off frequency of RF MEMS switches is 30 to 50 times better than their counterparts. They can be used in phase shifters and tunable circuits (matching networks, filters, etc.).

\section{SIMULATION RESULT}

As the actuation voltage increases the switch membrane is pulled towards the bottom electrode, there by resulting an increase in the capacitance.

The capacitance increases many times after the pull in as the switch membrane gets snapped to the lower electrode.

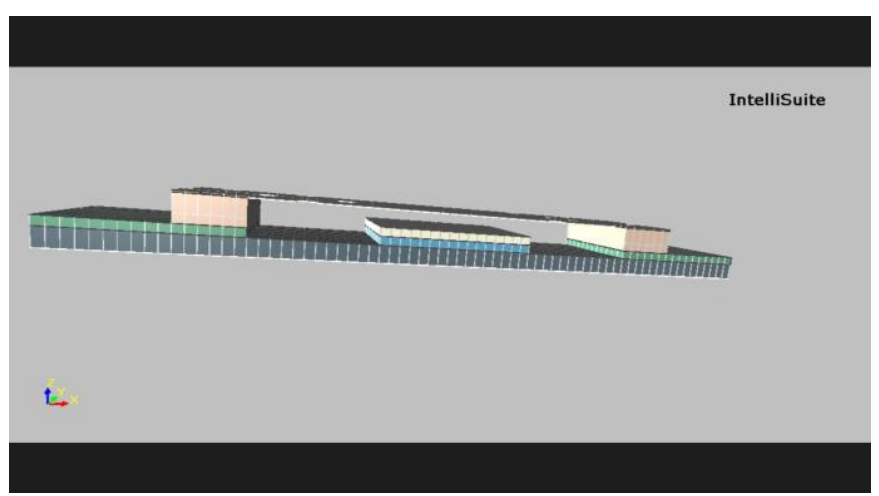

Fig 5 Up State Of Switch Designed Using Intellisuite

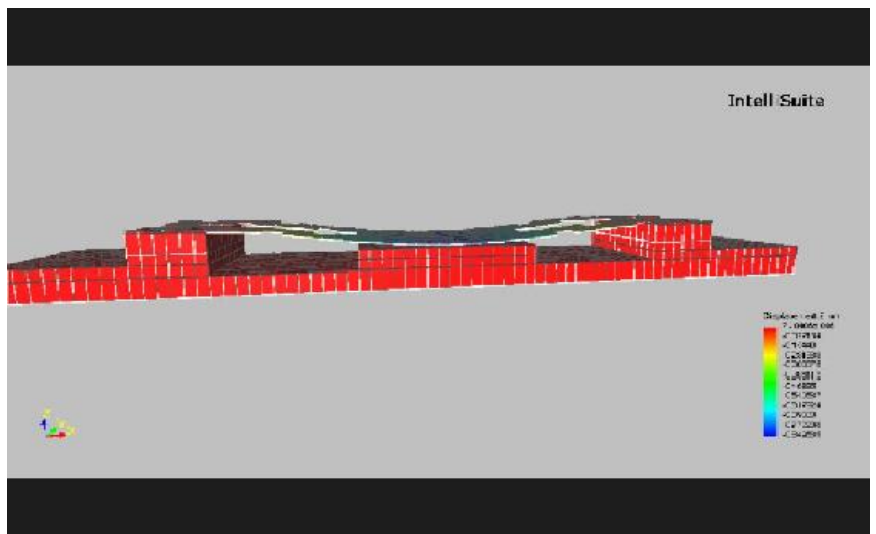

Fig 6 Down State Of Switch Designed Using Intellisuite.

Here we obtain 1 micro meter displacement at 0.35 voltage.

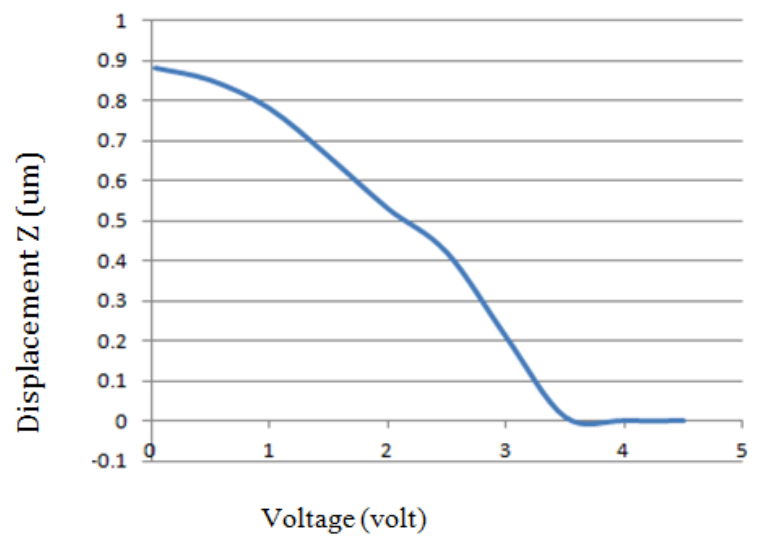

Fig 7 Pull In Characteristics Of RF MEMS Switch

\section{CONCLUSIONS}

RF MEMS capacitive shunt switch structure was designed and analyzed using 3DBuilder (which is the start-up module for Intellisuite). The design of this RF MEMS shunt switch using other modules like Thermo ElectroMechanical (TEM) and IntelliMask were also analyzed and designed.

Reconfiguring of antenna is achieved through changing its frequency, polarization or radiation characteristics by using Radio-Frequency Micro Electro Mechanical Systems (RF MEMS).

A low actuation voltage RF MEMS capacitive switch, suitable for achieving reconfigurable micro-machined antennas [8] is presented. A serpentine geometry for the flexures holding the switch membrane results in reducing the spring constant sufficiently low as to have a pull in voltage of $0.35 \mathrm{~V}$. 


\section{REFERENCES}

[1]. Chang won Jung, Ming-jer Lee, G. P. Li, and Franco De Flaviis, "Reconfigurable Scan-Beam Single-Arm Spiral Antenna Integrated With RF-MEMS Switches," IEEE Transactions on Antennas and Propagation, vol. 54, no. 2, February 2006.

[2]. Christos G Christodolou, Youssef Tawk,Steven A Lane and Scott R Erwin," Reconfigurable antennas for wireless and space applications ",Proceedings of the IEEE Vol 100 No .7 ,July 2012.

[3]. Greg H. Huff, and Jennifer T. Bernhard,"Integration of Packaged RF MEMS Switches With Radiation Pattern Reconfigurable Square Spiral Microstrip Antennas" IEEE Transactions on Antennas and Propagation, vol. 54, no. 2, february 2006.

[4]. Haslina Jaafar, Othman Sidek, Azman Miskam and Shukri Korakkottil," Design and Simulation of Microelectromechanical System Capacitive Shunt Switches," American J. of Engineering and Applied Sciences 2 (4): 655660, 2009 ,ISSN 1941-7020 @ 2009 Science Publications

[5]. Mingxin Song, " Design and analysis of a novel low actuation voltage capacitive RF MEMS switches", Proceedings of the 3rd IEEE International conference on Nano/Micro Engineered and Molecular systems, January ,2008

[6]. Chan, R. ; Dept. of Electr. \& Comput. Eng., Univ. of Illinois, Urbana, IL, USA ; Lesnick, R. ;Becher, D. ; Milton Feng Low-actuation voltage RF MEMS shunt switch with cold switching lifetime of seven billion cycles, Journal of Microelectromechanical Systems (JMEMS), oct 2003.

[7]. Muldavin, Jeremy B. Rebeiz, G.M. Inline "capacitive and DC-contact MEMS shunt switches", Microwave and Wireless components letters, Volume:11, Issue: 8,2001 , pp. 334 - 336

[8]. Farina, M. ; Keating, D. ; Jafri, I. ; Rozzi, T. "Full-wave analysis and electromechanical characterization of tunable capacitors", Antennas and Propagation Society International Symposium,2003.IEEE Volume: 1, pp. 737 - 740

\section{BIOGRAPHIE}

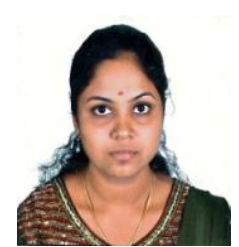

H. DIVYA received the B.E. degree in Electronics and Communication Engineering in the year 2012 and pursuing M.E degree in VLSI Design from Saveetha Engineering College. Her area of interests includes MEMS, VLSI Signal Processing and VLSI

Design. 